\title{
Real-world management of chronic myeloid leukemia in South Korea: the TARGET survey
}

\author{
Jieun Uhm ${ }^{1}$, Kyoung Ha Kim², Hyewon Lee ${ }^{3}$, Hawk Kim ${ }^{4}$, Slader Cassandra ${ }^{5}$, Inkyung Joo ${ }^{6}$, \\ Chul Won Jung ${ }^{7}$ \\ Division of Hematology Oncology, Department of Internal Medicine, ${ }^{1}$ Hanyang University Hospital, Hanyang University College of \\ Medicine, ${ }^{2}$ Soonchunhyang University College of Medicine, Seoul, ${ }^{3}$ Division of Tumor Immunology and Center for Hematologic \\ Malignancies, Research Institute and Hospital, National Cancer Center, Goyang, ${ }^{4}$ Division of Hematology, Gachon University Gil \\ Medical Center, Gachon University College of Medicine, Incheon, ${ }^{5}$ Novartis, Ltd., ${ }^{6}$ Novartis Korea, ${ }^{7}$ Division of Hematology-Oncology, \\ Department of Medicine, Samsung Medical Center, Sungkyunkwan University School of Medicine, Seoul, Korea
}

p-ISSN 2287-979X / e-ISSN 2288-0011 https://doi.org/10.5045/br.2021.2020260 Blood Res 2021;56:31-37.

Received on October 13, 2020

Revised on November 30, 2020

Accepted on December 17, 2020

*This study was supported by Novartis Korea, Ltd.

\section{Correspondence to}

Chul Won Jung, M.D., Ph.D.

Division of Hematology-Oncology,

Department of Medicine, Samsung Medical Center, Sungkyunkwan University School of Medicine, 81 Irwon-ro, Gangnam-gu, Seoul 06351, Korea

E-mail: leukemia1@skku.edu

(C) 2021 Korean Society of Hematology

\section{Background}

The global TARGET survey examined real-world management of chronic myeloid leukemia (CML) compared with international guideline recommendations. This report focused on the responses of physicians from South Korea compared with those of physicians from the rest of the world (ROW).

\section{Methods}

The self-administered, online survey, comprising 23 questions and clinical case scenarios, was completed between April and August 2017. It was designed to gather information on practicing physicians and local practices for CML diagnosis, disease monitoring, treatment, and adverse event (AE) management.

Results

While there were similarities in the mutation analysis and treatment efficacy between Korea and the ROW, there were also differences in CML management. Initial diagnostic testing was more comprehensive in Korea than in the ROW, and there was significantly better access to standardized polymerase chain reaction testing. Assessment of $B C R-A B L$ levels during the first 12 months of treatment was excellent in Korea, and there was greater frontline use of second-generation $B C R-A B L$ tyrosine kinase inhibitors. Korean physicians were significantly less likely to switch therapy for hematologic AEs. Treatment-free remission was not an important goal of therapy among Korean or ROW physicians.

\section{Conclusion}

This study identified some differences in the current CML management between Korea and the ROW; CML management in Korean patients was generally in line with the current guidelines.

Key Words Chronic myeloid leukemia, Management, Tyrosine kinase inhibitors, Real-life practice, Treatment-free remission

\section{INTRODUCTION}

The global incidence of chronic myeloid leukemia (CML), a myeloid malignancy driven by $B C R-A B L 1$ genetic rearrangement resulting in an oncoprotein with deregulated tyrosine kinase activity, ranges between 10 and 15 cases $/ 10 \%$ year [1-3]. In Korea, 458 CML cases were reported in 2012 , with a crude incidence of $0.91 / 10^{6}$; its incidence is currently increasing [4].

In line with international guidelines, $B C R-A B L$ tyrosine kinase inhibitors (TKIs) are recommended as the first-line treatment in Korean patients with CML [2, 5, 6]. Furthermore, imatinib, dasatinib, and nilotinib are reimbursable in Korea as first-line treatments [6]. Since the first introduction of imatinib in Korea in 2001, there has been a striking improvement in the survival of Korean patients with CML [4].

While international guidelines typically recommend eval- 
uation of the response to TKIs by monitoring blood parameters (hematologic response), cytogenetic monitoring of the $\mathrm{t}(9 ; 22)$ translocation (cytogenetic response), and polymerase chain reaction (PCR) testing for $B C R-A B L 1$ (molecular response), Korean guidelines favor molecular studies for treatment efficacy assessment over more invasive cytogenetic evaluations once the diagnosis is established [2, 5-7]. It is recommended that $B C R-A B L 1$ levels are monitored every 3 months during at least the first year of treatment and every 3-6 months thereafter [2, 5, 7]. The response rate and depth of response should also be monitored to detect non-compliance or therapy failure $[2,5,7]$.

Various reasons (e.g., technical, financial, and educational challenges) might prevent the implementation of international guidelines for CML management. A quantitative survey was then designed to collect general background information on TKI use in clinical practice to better understand real-world patient management during the chronic phase of CML. The aims of the multinational TARGET survey were to assess the current care of patients with CML in the participating countries compared with international guideline recommendations to identify challenges faced by physicians in implementing these recommendations and develop practical solutions to support physicians in optimizing CML management. In the analysis of the global results of the TARGET survey, several gaps in the current CML management were identified compared with international guidelines [8].

This report focused specifically on results among Korean physicians, comparing them with those among physicians from the rest of the world (ROW).

\section{MATERIALS AND METHODS}

The details of the survey methodology have been previously published [8]. In brief, the self-administered survey, available in English, Russian, Turkish, and Chinese, was completed online between April 1, 2017 and August 31, 2017. It included 23 questions divided into 7 sections and collected responding physicians' profile data and information regarding local practices for CML diagnosis, molecular monitoring and mutation analysis, treatment objectives, treatment efficacy and toxicities, and treatment discontinuation. Additionally, 7 hypothetical clinical cases were included, which helped examine physicians' responses to various scenarios of efficacy $(\mathrm{N}=2)$, toxicity $(\mathrm{N}=4)$, and treatment-free remission (TFR, $\mathrm{N}=1$ ).

\section{Statistical analysis}

The survey results were analyzed using the MODALISA software; the results were reported descriptively and considered significant when the $P$-value was $<0.05$, as calculated using the chi-squared test.

\section{RESULTS}

\section{Participants}

Among the 1,011 physicians contacted, 614 completed the questionnaire. Among these, 45 were from South Korea and 569 from the ROW. Those from the ROW comprised physicians from 32 countries in multiple regions worldwide (i.e., Africa, Asia-Pacific, Australia, Middle East, Russia, and Turkey), most commonly Russia ( $\mathrm{N}=115)$, China ( $\mathrm{N}=82$ ), India $(\mathrm{N}=72)$, and Turkey $(\mathrm{N}=49)$. Sixty-four percent of the Korean respondents were men, which was higher than the proportion of male respondents from the ROW (58\%). Generally, the Korean respondents had been treating CML for a lesser time than the ROW respondents. Among the Korean respondents, 30\% had been treating patients for 5-10 years and $52 \%$ for $>10$ years; among the ROW respondents, the corresponding rates were $20 \%$ and $69 \%$, respectively. Meanwhile, significantly more ROW respondents than Korean respondents had treated patients for $>15$ years $(46 \%$ vs. $32 \% ; P=0.052$ ). Additionally, the Korean physicians appeared to provide care for fewer patients than did the ROW physicians: $64 \%$ of the Korean respondents $(\mathrm{N}=43)$ personally provided care for 5-50 patients, whereas significantly more ROW respondents than Korean respondents had provided care for $50-100$ patients in the previous year $(22 \%$ vs. $7 \%$; $P=0.0008$ ).

The majority of the Korean respondents were administering first-line treatment (61.9\%), while $25.5 \%$ and $12.6 \%$ were administering second-line and third-line treatments, respectively; the results were generally similar with those for the ROW respondents. The most commonly administered first-, second-, and third-line treatments were imatinib/generic imatinib (39.5\%), dasatinib (33.8\%), and nilotinib (35.7\%), respectively, in Korea and imatinib/generic imatinib (79.4\%), nilotinib (38.4\%), and dasatinib (33.0\%), respectively, in the ROW. More Korean CML patients received second-generation $B C R-A B L$ TKIs, dasatinib, and nilotinib as first-line treatments than those in the ROW $(23.5 \%$ vs. $6.4 \%$ and $26.4 \%$ vs. $11.2 \%$, respectively).

\section{CML diagnosis}

Initial diagnostic testing appeared to be more comprehensive in Korea than in the ROW, with greater use of conventional karyotyping ( $89 \%$ vs. $78 \%$ ), quantitative PCR (qPCR; $89 \%$ vs. $74 \%$ ), qualitative PCR (61\% vs. $44 \%$ ), and fluorescence in situ hybridization (59\% vs. 39\%) and significantly lesser use of mutation analysis (2\% vs. $10 \%$; $P=0.09$ ). In contrast to that in the ROW, karyotyping in Korea was always performed on bone marrow aspirates (100\% vs. $94 \%$ ) but not on blood samples (0\% vs. $6 \%)$.

Both Korean and ROW physicians assessed patients' cardiovascular risk using echocardiography and/or electrocardiography (73\% vs. $72 \%$, respectively). The majority of the Korean and ROW respondents used the Framingham (31\% vs. $30 \%$ ) or EURO scores (38\% vs. $36 \%$ ) to assess the cardiovascular risk. While fasting cholesterol levels were 
measured to a similar degree in Korea and the ROW (73\% vs. $71 \%$ ), glucose levels were measured significantly more often in Korea than in the ROW (98\% vs. $88 \%$; $P=0.047$ ). Random glucose testing was performed significantly more often in Korea than in the ROW (26\% vs. $10 \%)$ and fasting glucose testing in the ROW than in Korea (60\% vs. $35 \%$; $P=0.0007)$.

\section{Molecular monitoring and mutation analysis}

The type of established conversion factor to standardized PCR was different between Korea and the ROW. In Korea, $32 \%$ used an international reference laboratory, and $7 \%$ used a national reference laboratory; in the ROW, the corresponding rates were $40 \%$ and $17 \%$, respectively. Commercial standards were used significantly more often in Korea than in the ROW ( $48 \%$ vs. $16 \% ; P<0.0001$ ). The majority of the Korean and ROW respondents (61\%) were unaware of when the last standardization had occurred $(61 \%$ and $52 \%$, respectively).

The molecular monitoring frequency in Korea was significantly better than that in the ROW ( $P=0.047)$ : $98 \%$ of the Korean respondents knew that $B C R-A B L$ levels should be assessed every 3 months within the first 12 months of treatment, and this was achieved in clinical practice in $93 \%$ of cases. Conversely, $88 \%$ of the ROW respondents knew that $B C R-A B L$ levels should be assessed every 3 months within the first 12 months of treatment; however, this was only achieved in $48 \%$ of cases. Cost and laboratory capability were less often cited as barriers to testing in Korea than in the ROW ( $37 \%$ vs. $49 \%$ and $5 \%$ vs. $10 \%$, respectively).

According to 42 Korean respondents, the majority of laboratory reports included information regarding $B C R-A B L$ copy number $(93 \%)$, assay type $(88 \%)$, international reporting scale results (82\%), and control gene copy number (82\%). Slightly fewer reports contained raw results (71\%) and trends over time $(36 \%)$, although raw results were included in the reports significantly more often in Korea than in the ROW (71\% vs. $46 \% ; P=0.001$ ).

Mutation status was requested by similar proportions of the Korean and ROW respondents for treatment failure as- sessment, excluding tolerance ( $86 \%$ vs. $85 \%$ ), any sign of loss of hematologic or cytogenetic response ( $80 \%$ vs. $82 \%)$, progression to the accelerated or blast phase ( $80 \%$ vs. $77 \%)$, and significant $B C R-A B L$ level increase from 0.001 to 0.01 (1-log increase) after achieving a major molecular response (MMR, 61\% vs. 50\%).

\section{Treatment objectives}

TFR was considered by a few Korean respondents (8\%) as their primary treatment goal (Fig. 1). MMR achievement was the most important treatment objective (30\%), followed by overall survival prolongation (18\%), early molecular response (EMR) achievement (13\%), or complete cytogenetic response achievement (CCyR, 13\%). The priorities of the ROW respondents differed from those of the Korean respondents, with EMR achievement (29\%) as the most important treatment objective, followed by overall survival prolongation (14\%) and MMR achievement (13\%). Only 6\% of the ROW respondents considered TFR as their primary treatment goal.

TFR was considered a relatively less important factor influencing treatment decisions among the Korean respondents (13\%), while the presence of comorbidities (40\%), Sokal/ European Treatment and Outcome Study (EUTOS)/EUTOS long-term survival (ELTS) score (15\%), and patient age (15\%) were considered as more important. The Sokal, EUTOS, or ELTS score and patient preference were considered more often when making treatment decisions by the ROW respondents than by the Korean respondents (36\% vs. $15 \%$ and $12 \%$ vs. $5 \%$, respectively).

The majority of the Korean physicians (64\%) would always select a second-generation TKI over imatinib as frontline treatment for patients with chronic-phase CML compared with only $19 \%$ of the ROW physicians $(P<0.0001)$. A second-generation TKI would more often be selected over imatinib as frontline treatment for a patient with chronic-phase CML if TFR is a high-priority goal for the patient $(51 \%$ vs. $38 \%)$ or if the patient has a high Sokal risk score $(68 \%$ vs. 29\%) among the ROW respondents than among the Korean respondents.

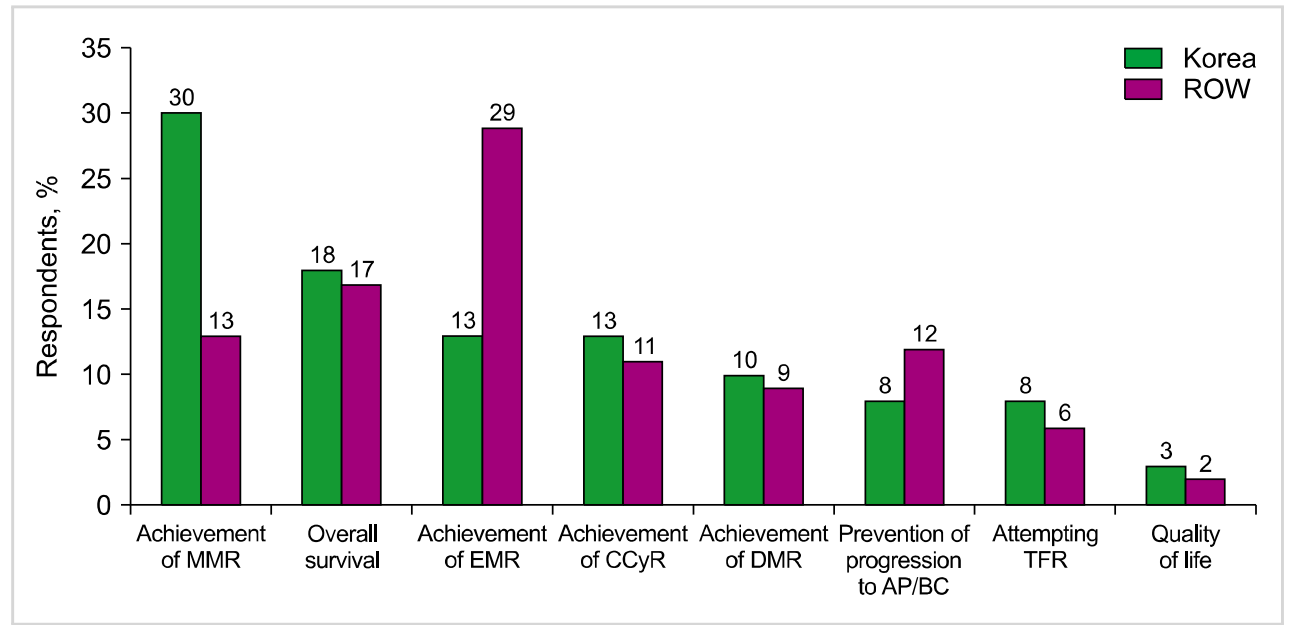

Fig. 1. Most important first-line treatment objectives. The respondents assigned a priority from 1 (most important) to 8 (least important) for the listed first-line treatment objectives.

Abbreviations: AP, accelerated phase; $\mathrm{BC}$, blast crisis; CCyR, complete cytogenetic response; DMR, deep molecular response; EMR, early molecular response; MMR, major molecular response; ROW, rest of the world; TFR, treatment-free remission. 


\section{Treatment efficacy}

Among the Korean respondents, the typical molecular response rates in Korean patients treated with first-line imatinib were $13 \%$ for EMR, 19\% for CCyR at 6 months, $26 \%$ for MMR at 12 months, and 22\% for MMR after 12 months. In addition, $6 \%$ of Korean patients never achieved an MMR, and $14 \%$ are currently on MR4.5. The results were generally similar for the ROW.

To assess the interpretation of the physicians of the European LeukemiaNet (ELN) guidelines [7], they were asked how they would respond to certain hypothetical $B C R-A B L$ scenarios in clinical practice. In most situations of suboptimal responses (i.e., $B C R-A B L$ level of $>10 \%$ at $3 \mathrm{mo} ; B C R-A B L$ level of $1-10 \%$ at $6 \mathrm{mo}$; and $B C R-A B L$ level of $0.1-1 \%$ at 12 mo [7]), the majority of the Korean respondents (59-78\%) considered these as suboptimal responses, and approximately two-thirds (61-66\%) stated that they would re-evaluate the patient in 30-60 days, with only $2-5 \%$ choosing to immediately switch treatment (Table 1 ). Regarding the scenario of $B C R-A B L$ level of $<0.1 \%$ but with residual disease at 24 months, only $24 \%$ of the Korean respondents considered this a suboptimal response, while $37 \%$ and $34 \%$ considered it an acceptable or optimal response, respectively; $27 \%$ chose to re-evaluate in 30-60 days (Table 1). The majority (66\%) of the physicians chose not to switch treatment, and only $7 \%$ opted to switch immediately. The scenario reflects optimal response according to the ELN 2013 guidelines [7]; however, for patients who wish to achieve TFR, the response may be considered suboptimal. Significantly more Korean respondents than ROW respondents considered this an optimal response ( $34 \%$ vs. $14 \% ; P=0.0006)$.

In 2 situations of ELN-defined treatment failure (i.e., $B C R-A B L$ level of $>10 \%$ at $6 \mathrm{mo}$ and $B C R-A B L$ level of $>1 \%$ at $12 \mathrm{mo}$ ), the majority of the Korean respondents recognized these as treatment failure $(66-71 \%)$ and chose to switch treatment immediately (73-76\%) (Table 1).

\section{Treatment-related toxicities}

Zero and two percent of the Korean respondents stated that they would change treatment for persistent grade 1 hematologic and non-hematologic adverse events (AEs), respectively. Contrastingly, $49 \%$ and $95 \%$ of the Korean respondents stated that they would do so for grade $3 / 4$ hematologic and non-hematologic AEs, respectively. In the case of persistent grade $2 \mathrm{AEs}$, 9\% of the Korean respondents would switch for hematologic AEs and 42\% for non-hematologic AEs. Conversely, there were significant differences found among the ROW respondents; significantly more ROW respondents than Korean respondents switched therapy for grade $3 / 4$ (84\% vs. $49 \%)$ and persistent grade 2 hematologic AEs (33\% vs. 9\%; $P<0.0001)$.

\section{Treatment discontinuation}

The Korean respondents were significantly more confident that their local laboratory met the testing criteria for monitoring TFR in patients than were the ROW respondents.

Table 1. Physicians' responses regarding how hypothetical patients receiving first-line treatment for chronic-phase CML would be managed based on $B C R-A B L$ levels.

\begin{tabular}{|c|c|c|c|}
\hline \multirow{2}{*}{$\%$ of respondents } & \multicolumn{3}{|c|}{ Physicians' response $(\mathrm{N}=41)$} \\
\hline & Immediately switch & Re-evaluate in $30-60$ days & Will not switch \\
\hline \multicolumn{4}{|l|}{ ELN-defined optimal response [7] } \\
\hline \multicolumn{4}{|c|}{$B C R-A B L$ level of $<0.1 \%$ with residual disease at $24 \mathrm{mo}$} \\
\hline Korea & 7 & 27 & 66 \\
\hline ROW & 18 & 28 & 53 \\
\hline \multicolumn{4}{|l|}{ ELN-defined suboptimal response [7] } \\
\hline \multicolumn{4}{|l|}{$B C R-A B L$ level of $>10 \%$ at $3 \mathrm{mo}$} \\
\hline Korea & 5 & 66 & 29 \\
\hline ROW & 15 & 55 & 29 \\
\hline \multicolumn{4}{|l|}{$B C R-A B L$ level of $1-10 \%$ at $6 \mathrm{mo}$} \\
\hline Korea & 2 & 61 & 37 \\
\hline ROW & 13 & 51 & 35 \\
\hline \multicolumn{4}{|l|}{$B C R-A B L$ level of $0.1-1 \%$ at $12 \mathrm{mo}$} \\
\hline Korea & 2 & 63 & 34 \\
\hline ROW & 14 & 52 & 33 \\
\hline \multicolumn{4}{|l|}{ ELN-defined treatment failure [7] } \\
\hline \multicolumn{4}{|l|}{$B C R-A B L$ level of $>10 \%$ at $6 \mathrm{mo}$} \\
\hline Korea & 76 & 15 & 10 \\
\hline ROW & 76 & 20 & 4 \\
\hline \multicolumn{4}{|l|}{$B C R-A B L$ level of $>1 \%$ at $12 \mathrm{mo}$} \\
\hline Korea & 73 & 17 & 10 \\
\hline ROW & 74 & 20 & 6 \\
\hline
\end{tabular}

Abbreviations: CML, chronic myeloid leukemia; ELN, European LeukemiaNet; ROW, rest of the world. 
The mean compliance scores (with 0 indicating "not at all" and 5 indicating "full compliance") among the Korean respondents for capacity to test patients every 4-6 weeks, qPCR test result turnaround within 4 weeks, and capability to perform rapid intervention if a patient needs to restart therapy were $4.6,4.4$, and 4.4 , respectively, compared with 3.4 , 3.7, and 3.6 among the ROW respondents.

In line with currently available data, deep molecular response (DMR) achievement as a prerequisite for TFR was recognized by only $28 \%$ of the Korean physicians compared with $49 \%$ of the ROW physicians (Fig. 2). Instead, the Korean physicians considered the duration of DMR as the most important factor for attempting TFR (36\% vs. $19 \%$ of the ROW respondents) [9].

The major hurdles for attempting TFR in current practice in Korea were a lack of long-term data (33\%) and an insufficient molecular monitoring capability (frequency and/or sensitivity, 23\%), e.g., PCR test turnaround time and PCR sensitivity to a deeper molecular response, followed by financial burden (18\%) and a lack of guidelines (15\%). In contrast, more ROW physicians cited a lack of guidelines (31\%), and fewer cited a lack of long-term data $(10 \%)$ as the major hurdles. A lack of personal experience ( $5 \%$ vs. $6 \%$ ), patients' concerns $(5 \%$ vs. $10 \%)$, and consequences at relapse $(0 \%$ vs. $8 \%$ ) were considered less important factors among the Korean ROW physicians.

\section{Clinical cases}

In the case of a patient with an optimal response (MMR at $12 \mathrm{mo}$ ) but not in a DMR at 24 months, only $9 \%$ of the Korean respondents would change the patient's treatment compared with $21 \%$ of the ROW respondents. Management of a patient with a suboptimal response at 3 months (i.e., did not achieve EMR) yielded similar responses, with $63 \%$ and $52 \%$ of the Korean and ROW respondents choosing to follow the ELN guideline's watch and wait recommendation, and $37 \%$ and $48 \%$ opting to adjust treatment, respectively [7].

Similar proportions of the Korean and ROW respondents would use granulocyte colony-stimulating factor to treat grade 3 neutropenia in a patient with an intermediate Sokal risk and an optimal response with first-line treatment with a second-generation TKI ( $41 \%$ vs. $46 \%$ ). Upon spontaneous resolution within 2 weeks, $71 \%$ and $57 \%$ of the Korean and ROW physicians stated that they would restart TKI treatment at the same dose, while $29 \%$ and $38 \%$ would restart at a reduced dose, respectively. After 6 months and with optimal response, similar proportions of the Korean and ROW physicians opted to continue treatment despite the patient experiencing grade 2 increase in the bilirubin level (68\% vs. $74 \%$, respectively). At 12 months and with achievement of MMR, significantly more Korean physicians than ROW physicians would maintain the dose but initiate statin treatment for a steady increase in the cholesterol level (90\% vs. $72 \% ; P=0.01$ ).

The responses were split in the case of persistent grade 2 muscle cramps in a patient receiving imatinib $400 \mathrm{mg} /$ day with an optimal response at 6 months, with significantly more Korean respondents than ROW respondents choosing to reduce the imatinib dose $(41 \%$ vs. $20 \% ; P<0.0001)$. Meanwhile, more ROW physicians stated that they would continue imatinib at the same dose (40\% vs. $29 \%)$ or switch to a second-generation TKI (40\% vs. $29 \%$ ).

In the case of a grade 3 rash in a patient treated with a second-generation TKI for 3 months, $73 \%$ of the Korean respondents would interrupt TKI administration and start treatment for skin rash, while $27 \%$ would reduce the TKI dose and treat the rash. The corresponding results for the ROW respondents were $62 \%$ and $38 \%$, respectively. There were differences found in the treatment for rash between the Korean and ROW respondents, with a short course of systemic steroid being used significantly more often in Korea (63\% vs. $47 \%$; $P<0.001$ ). Other popular choices included the use of a systemic antihistamine (24\% vs. $31 \%)$ and local therapies, such as lotion or glucocorticoid therapy (12\% vs. $22 \%)$.

The 6th case helped examine the physicians' responses to the management of a 31-year-old female patient who

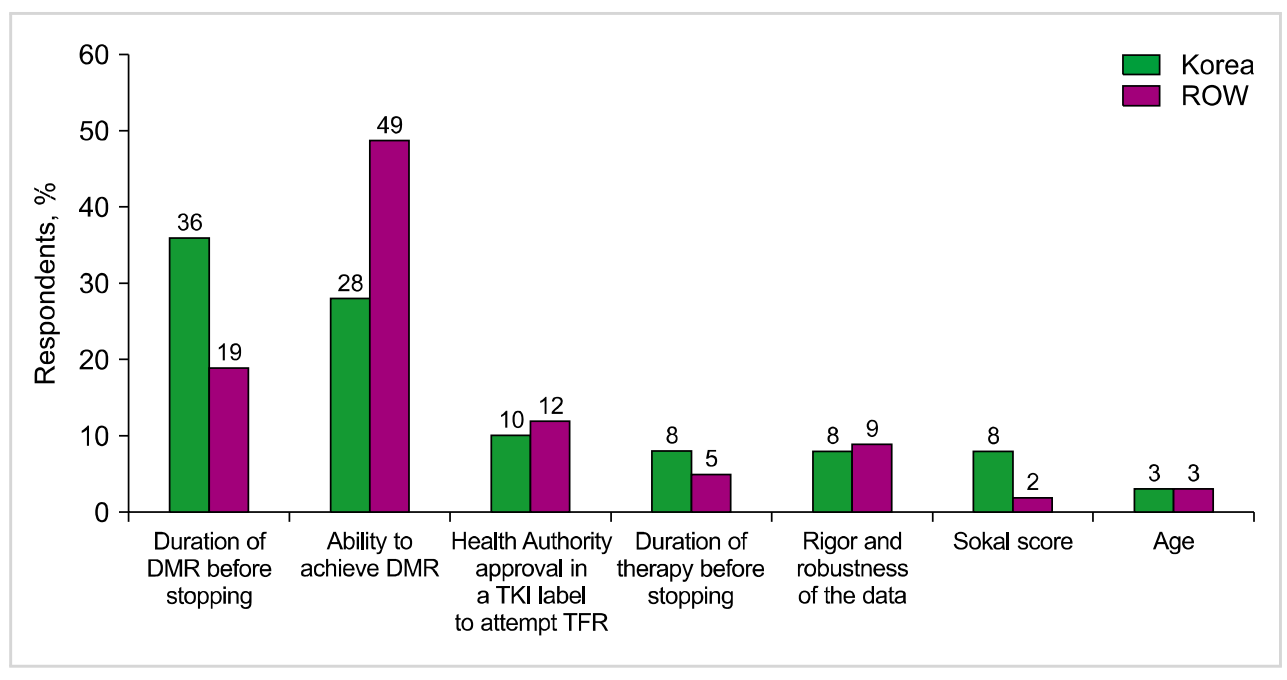

Fig. 2. Most important factors for attempting TFR. Each of the respondents assigned a priority from 1 (most important) to 7 (least important).

Abbreviations: DMR, deep molecular response; ROW, rest of the world; TFR, treatment-free remission; TKI, tyrosine kinase inhibitor. 
achieved MR4 2 years after switching from imatinib to nilotinib. After maintaining MR4 for a further 12 months, the patient was keen to achieve TFR to allow her to start a family. Among the Korean respondents, $15 \%$ would attempt TFR now, while the majority would wait until MR4.5 is achieved and maintained for $12(15 \%)$ or 24 months (54\%). Fifteen percent of the Korean physicians would not attempt TFR because they were not assured of its safety. The responses were broadly similar among the ROW respondents, except that significantly more would not attempt TFR owing to a lack of necessary conditions $(8 \%$ vs. $0 \%$ of the Korean respondents; $P=0.03$ ).

The final case helped examine the treatment options in a patient with MMR experiencing pleural effusion (not requiring oxygen) after 36 months of treatment with dasatinib. In addition to stopping dasatinib, the treatment choices among the Korean physicians included diuretics (71\%), glucocorticoid $(63 \%)$, thoracentesis $(22 \%)$, and chest computed tomography $(17 \%)$. Thoracentesis $(35 \%)$, computed tomography $(33 \%)$, and chest tube insertion $(7 \%)$ were more common among the ROW respondents.

\section{DISCUSSION}

The TARGET survey confirmed that the current CML management in Korea is largely in line with the recommendations of the international and Korean guidelines [5-7]. While there were similarities for the mutation analysis and treatment efficacy between Korea and the ROW, there were also quite a few differences in CML management. Initial diagnostic testing appeared to be more comprehensive in Korea than in the ROW, and most centers in Korea were able to access standardized PCR (86\%) mainly by conversion factor from commercialized standards followed by the use of an international reference laboratory compared with those in the ROW [fewer centers were able to access standardized PCR (73\%), with lesser use of commercialized standards]. $B C R-A B L$ level assessment during the first 12 months of treatment was excellent in Korea, with almost all Korean patients monitored every 3 months, compared with less than half of ROW patients. Response monitoring during the first 12 months of treatment was extremely high, with MMR being cited most often as the main treatment objective.

The treatment patterns also differed between Korea and the ROW, with greater frontline use of second-generation TKIs and greater preference of original imatinib to generic imatinib, undoubtedly because imatinib, dasatinib, and nilotinib are all reimbursable as first-line treatments in Korea [6]. For a patient with a suboptimal response, the majority of the Korean physicians decided to monitor $B C R-A B L$ levels closely rather than to change the TKI immediately. Comorbidities, the Sokal/EUTOS/ELTS scores, and patient age were considered as the most important factors in choosing TKIs among the Korean physicians. The real-world practice of Korean physicians is closely aligned with the Korean guideline that covers all aspects of CML management (from diagnosis to treatment) and is published in Korean so that all physicians in Korea who treat patients with CML can access and refer to it [6].

Finally, the management of some toxicities differed between the Korean and ROW physicians, with the former significantly less likely to switch therapy owing to hematologic AEs. The Korean physicians tended to maintain TKI doses with significant hematologic AEs if they are resolved within 2 weeks. However, if a patient develops persistent grade 2 non-hematologic AEs, such as hyperbilirubinemia, muscle cramps, and fluid retention, except metabolic AEs, more Korean physicians chose to reduce the TKI dose to manage AEs. For metabolic AEs, such as hypercholesterolemia, almost all Korean physicians initiated medical intervention while maintaining the TKI dose. Generally, the Korean physicians followed the Korean guideline to manage toxicities and optimize the dose of TKIs to obtain and monitor the optimal response [6].

As the Korean guidelines were published in 2015, the guidelines did not include TFR, although EMR and DMR were included [6]. An interesting finding is that although there was no mention of TFR in the 2015 Korean guidelines, many Korean physicians were aware of TFR and attempted to implement TFR in the case presented in the survey, including understanding the importance of achieving a DMR and its duration. As long-term data accumulation could change the practice guidelines, Korean physicians have awaited long-term data to attempt TFR with safety. This could be another reason why the Korean physicians would prefer second-generation TKIs to imatinib as the first-line treatment for chronic-phase CML.

These real-world data collected from a total of 614 practicing hematologists, including 45 physicians from South Korea, represent a particular strength of the study, providing useful information on the local delivery of care. As the survey was conducted in 4 different languages, and an English survey was available to Korean respondents, there might be a slight difference in the answers to some questions compared to the answers to the survey in its mother language. However, it is unlikely that this had a major impact on the survey results.

\section{CONCLUSIONS}

The TARGET survey identified some differences in the current CML management between Korea and the ROW; CML management in Korean patients was generally in line with the current guidelines.

\section{ACKNOWLEDGMENTS}

This study was funded by Novartis. Medical writing assistance with the preparation of this manuscript was provided by Solenn Le Clanche from Galien Health Publishing and KPL. 


\section{Authors' Disclosures of Potential Conflicts of Interest}

No potential conflicts of interest relevant to this article were reported.

\section{REFERENCES}

1. O'Brien S, Radich JP, Abboud CN, et al. Chronic myelogenous leukemia, version 1.2015. J Natl Compr Canc Netw 2014;12: 1590-610.

2. Hochhaus A, Saussele S, Rosti G, et al. Chronic myeloid leukaemia: ESMO Clinical Practice Guidelines for diagnosis, treatment and follow-up. Ann Oncol 2017;28(Suppl 4):iv41-51.

3. Holyoake TL, Vetrie D. The chronic myeloid leukemia stem cell: stemming the tide of persistence. Blood 2017;129:1595-606.

4. Park EH, Lee H, Won YJ, et al. Nationwide statistical analysis of myeloid malignancies in Korea: incidence and survival rate from
1999 to 2012. Blood Res 2015;50:204-17.

5. National Comprehensive Cancer Network. NCCN Practice Guidelines in Oncology: chronic myeloid leukemia. Version 4. Plymouth Meeting, PA: National Comprehensive Cancer Network, 2018. (Accessed March 29, 2018, at https://www.nccn. org/professionals/physician_gls/pdf/cml.pdf).

6. Kim DY, Lee JO, Kim KH, et al. Korean guidelines for treating chronic myelogenous leukemia-The Korean Society of Hematology Chronic Myelogenous Leukemia Working Party. Korean J Med 2015;88:406-19.

7. Baccarani M, Deininger MW, Rosti G, et al. European LeukemiaNet recommendations for the management of chronic myeloid leukemia: 2013. Blood 2013;122:872-84.

8. Turkina A, Wang J, Mathews V, et al. TARGET: a survey of real-world management of chronic myeloid leukaemia across 33 countries. Br J Haematol 2020;190:869-76.

9. Gale RP, Hochhaus A. Therapy-free remission in chronic myeloid leukemia: possible mechanism. Expert Rev Hematol 2018;11: 269-72. 